\title{
La profession d'enseignant de français langue étrangère au Monténégro: le rapport à la formation et les difficultés du métier
}

\author{
IVONA JOVANOVIĆ \\ Université du Monténégro
}

Received: 23 April 2016/ Accepted: 13 February 2017

ISSN: $1698-7467$

\begin{abstract}
RÉSUMÉ: Suite à une présentation de l'état des lieux de l'enseignement de FLE au Monténégro, cette contribution analyse les difficultés des enseignants monténégrins dans leur voie professionnelle et propose de réfléchir à un réaménagement de leur cursus de formation initiale. Le travail repose d'une part sur des données recueillies auprès des enseignants de FLE actifs au Monténégro et d'autre part sur les données relevées auprès des élèves provenant d'écoles situées dans les localités où l'enseignement du français a connu une régression considérable.
\end{abstract}

Mots clés: Enseignant, FLE, Monténégro, formation, difficultés

Profession of a teacher of French as a foreign language in Montenegro: attitude to education and difficulties of the profession

\begin{abstract}
After the presentation of the situation which refers to studying French language in Montenegro, this paper analyzes the difficulties encountered by Montenegrin teachers on their career path and suggests changes in the curricula of undergraduate studies. The paper is based on the data provided by currently active French teachers in Montenegro and on the other hand on the data provided by the students of those schools which are in places in which the interest in learning French has considerably decreased.
\end{abstract}

Keywords: Teacher, French as a foreign language, Montenegro, education, difficulties

\section{InTRODUCTION}

Le fait d'enseigner les langues remonte à l'Antiquité, mais la création d'une profession d'enseignant de langues est un travail toujours en chantier (Kelly, 2014 : 400). Cependant, selon Jean Louis Chiss, la nature et la modalité de la formation des enseignants de langues dépendent largement du statut sociolinguistique de la langue enseignée et des politiques linguistiques et éducatives du pays (Chiss, 2007:186).

Le Monténégro, le plus petit pays des Balkans, a renouvelé son indépendance en 2006. Francophile et francophone dans le passé, il l'est beaucoup moins de nos jours, sous la pression de l'anglais d'une part, mais également du russe et de l'italien dont le poids historique se ressent toujours. Dans ce contexte, l'enseignement du FLE a été réduit et les enseignants se tournent davantage vers d'autres métiers. On constate depuis une dizaine d'années une forte augmentation de touristes francophones et de ce fait un besoin croissant de professionnels du tourisme maîtrisant la langue française. 
Le cadre universitaire dans lequel s'inscrit la formation initiale des enseignants de FLE est en pleine reconstruction et s'apprête à être soumis à une évaluation externe en 2017. L'organisation sur laquelle repose la maquette actuelle s'inspire de celle du département de Langue et littérature françaises de la Faculté de philologie de l'Université de Belgrade à laquelle elle a emprunté sa structure de base dans les années qui ont précédé l'indépendance du pays. Ses contenus en vigueur insistent davantage sur l'enseignement de la linguistique et de la littérature, et beaucoup moins sur les activités langagières visant le développement des capacités communicatives des futurs enseignants (Lee, 2011, 133).

Au sein des écoles monténégrines, l'enseignant de FLE semble être esseulé. Il est généralement le seul enseignant de FLE dans son établissement et la langue qu'il doit transmettre est souvent ressentie comme quelque chose de difficile à prononcer et complexe à apprendre. Les effectifs élevés des classes dans lesquelles il enseigne, une discipline de plus en plus difficile à maintenir et l'hétérogénéité des groupes lui posent également de sérieux problèmes.

A la suite d'une présentation de l'état des lieux de l'enseignement du FLE au Monténégro, cet article expose la problématique de la formation des enseignants de FLE et celle des difficultés rencontrées dans leurs milieux professionnels. Il présente l'analyse des données recueillies auprès des enseignants de FLE monténégrins à l'égard de leur formation et de leur profession et propose un réajustement des programmes de leur formation initiale afin de les rendre plus motivés, plus performants et plus aptes à affronter les difficultés du métier.

\section{L'ENSEIGNEMENT DE FLE AU MONTÉNÉGRO}

L'enseignement institutionnel de la langue française au Monténégro ${ }^{1}$ remonte à 1869 à l'époque de l'ouverture du prestigieux Institut de jeunes filles de l'Impératrice Marie dans la minuscule capitale monténégrine Cetinje. Dans cette institution financée par la Russie, hormis le russe, la primauté fut accordée à l'apprentissage du français, enseigné dans la plupart des cas par des institutrices de nationalité française qui insistaient pour que leurs pensionnaires parlent français en dehors des classes de langue (Jovanović, 2016 : 235). Le français fut la première langue étrangère enseignée dans le premier lycée d'État ouvert dans la même ville en 1880 .

Dans la période entre les deux guerres, sur le territoire monténégrin qui fut incorporé en 1918 au Royaume des Serbes, Croates et Slovènes, le français a été enseigné en tant que première langue étrangère dans tous les lycées, et cela de la première à la dernière année (pendant 8 ans à raison de 3 heures par semaine). ${ }^{2}$ La discipline est enseignée par la méthode directe qui favorisait l'utilisation de la langue orale et accordait une importance particulière à la prononciation (Laval, 1920 :157). Cetinje disposait même à l'époque d'un jardin d'enfants dirigé par une Française (Čakić, 1997 : 9).

Après la deuxième guerre mondiale, dans la plus petite des six républiques de la Yougoslavie socialiste, le français maintient jusqu'aux années 1970 une position privilégiée par rapport aux autres langues étrangères. Il continuera d'être enseigné dans la plupart des collèges et lycées monténégrins comme première langue étrangère. L'introduction du russe

${ }^{1}$ Le Monténégro d'autrefois (le Vieux Monténégro) était constitué de la bourgade de Cetinje et de ses alentours (sans le littoral, la capitale actuelle Podgorica et le nord du pays).

${ }^{2}$ Nastavni planovi i programi, Glasnik JPD, knj.XV, sv.4, 1934, p.345 et 346. 
et de l'anglais dans les classes de langues ne sera proposé qu'en 1960 par le Conseil de l'Education nationale de la République populaire du Monténégro (Bokan, 1961 : 2). Toutefois, vu le manque de professeurs diplômés, ce sont les enseignants de FLE qui garderont encore un certain temps la primauté dans les classes de langues étrangères (Kapetanović, 1961 : 3). Puis, peu à peu, l'anglais prendra de plus en plus d'ampleur jusqu'à être pratiquement devenu, depuis deux décennies, la seule première langue étrangère enseignée dans les institutions scolaires du pays.

Ce n'est qu'à la suite de la désintégration de la Yougoslavie, plus précisément en 1992, que le ministère de l'Education du Monténégro a inscrit dans les programmes d'enseignement primaire une deuxième langue étrangère obligatoire, et cela à partir de la 7ème classe (âge de 13 ans). ${ }^{3}$ Enseignée à raison de trois heures par semaine, la deuxième langue étrangère jouit de nos jours du statut de discipline obligatoire dans les écoles primaires et dans les lycées d'enseignement général. Selon le choix des élèves, l'allemand, le français, l'italien et le russe sont les langues les plus souvent proposées. Il est toutefois possible, dans les établissements scolaires disposant d'enseignants formés, de suivre de manière facultative des cours de deuxième langue étrangère avant d'avoir accédé en classe de $7^{\text {ème }}$, ou des cours de troisième langue étrangère dans les lycées.

Cependant, le choix des deuxième et troisième langues étrangères enseignées dans les établissements scolaires au Monténégro ne dépend pas seulement du désir des enfants et des ressources humaines disponibles, mais aussi de la volonté des chefs d'établissements, dont le recrutement est en grande partie influencé par le parti politique au pouvoir. Dans les écoles situées essentiellement dans le nord du pays, là où la population est restée liée à la Serbie voisine, c'est le russe, traditionnellement favorisé, qui est la deuxième langue étrangère la plus couramment enseignée (Jovanović, 2007 : 897). Le français y a gardé une position non négligeable en étant offert aux apprenants dans un certain nombre d'écoles. D'autre part, certaines régions habitées par une population essentiellement musulmane (villes de Plav, Rožaje, Gusinje) favorisent l'apprentissage du français, semblerait-il pour des raisons plus pratiques, étant donné qu'un pourcentage relativement élevé de la population de la région a immigré au Luxembourg.

En revanche, dans les établissements scolaires qui se situent dans les régions les plus développées du pays, sur la côte et dans la capitale Podgorica, c'est surtout l'italien que les élèves favorisent. L'italien a la réputation d'être une langue mélodieuse beaucoup plus facile à apprendre que le français ou de l'allemand. Mentionnons également que hormis la proximité géographique, la popularité de l'italien dans le sud a de fortes raisons historiques puisque le littoral a fait partie pendant quatre siècles de la République de Venise. De ce fait, et en particulier dans la région des Bouches de Kotor, l'italien demeure une langue seconde pour la majorité de la population.

Par ailleurs, dans l'esprit d'un certain nombre de personnes résidant dans le noyau historique du pays (Cetinje et ses alentours), l'image négative du français est en partie liée à la politique de la France, favorable après la Première guerre mondiale à l'unification du Monténégro à la Serbie. Depuis la reconnaissance de l'indépendance du pays en 2006 et le réveil du sentiment national chez la population, cette idée d'injustice envers le Monténégro,

\footnotetext{
${ }^{3}$ L'enseignement primaire s'étend à neuf ans dans le système scolaire monténégrin et est réparti en trois cycles. La septième classe correspond à la première année du dernier cycle d'enseignement primaire.
} 
causée en partie par la politique de la France d'autrefois, semblerait plus prononcé. Il s'avère qu'en revanche, l'Italie avait soutenue davantage la restauration du Monténégro, en ayant mis sur pied dans la petite localité de Gaète, entre Rome et Naples, une armée monténégrine luttant pour l'indépendance usurpé (Andrijašević, 2000 : 167).

On constate que le français, si enseigné au Monténégro autrefois, figure parmi les langues les moins attrayantes à étudier de nos jours, suivi par l'allemand pour lequel les apprenants montrent actuellement encore moins d'intérêt. Cependant, ces dernières années, l'enseignement de l'allemand prend de plus en plus d'ampleur et sa popularité est en hausse. Par ailleurs, les ambassades d'Allemagne et d'Autriche au Monténégro financent des enseignants manquants, attribuent régulièrement des bourses aux étudiants et soutiennent les écoles et les départements universitaires en matériel technique et informatique.

Tableau I: Effectifs d'élèves dans les classes de langue étrangère dans les écoles primaires et secondaires au Monténégro pour l'année scolaire 2011/2012.

(Source: ministère de l'Éducation nationale - secteur pour les Technologies de l'information et de la communication)

Ecole primaire

\begin{tabular}{|c|c|c|c|c|c|}
\hline $\begin{array}{c}\text { Matière } \\
\text { obligatoire }\end{array}$ & $\begin{array}{c}\text { Matière } \\
\text { obligatoire }\end{array}$ & $\begin{array}{c}\text { Matière } \\
\text { obligatoire }\end{array}$ & \\
\hline Anglais & 42926 & Anglais & 9613 & Anglais & 14932 \\
\hline Russe & 1723 & Italien & 3323 & Russe & 1706 \\
\hline Italien & 1570 & Russe & 2924 & Italien & 701 \\
\hline Français & 928 & Français & 1900 & Français & 432 \\
\hline Allemand & 198 & Allemand & 997 & Allemand & 455 \\
\hline $\begin{array}{c}\text { Matière } \\
\text { facultative }\end{array}$ & 5958 & $\begin{array}{c}\text { Matière } \\
\text { facultative }\end{array}$ & $\begin{array}{c}\text { Matière } \\
\text { facultative }\end{array}$ & \\
\hline Russe & 5711 & Espagnol & 457 & Anglais & 705 \\
\hline Italien & 3775 & Français & 322 & Russe & 276 \\
\hline Français & 1698 & Turque & 199 & Français & 250 \\
\hline Allemand & 371 & Russe & 136 & Allemand & 120 \\
\hline Anglais & 152 & & & & \\
\hline Espagnol & & & & \\
\hline
\end{tabular}

L'Institut français, installé depuis 1997 dans la capitale monténégrine Podgorica, organise régulièrement des ateliers, des conférences, ainsi que d'autres évènements culturels auxquels les enseignants de FLE aiment prendre part. Il offre également au public la possibilité de suivre des cours de FLE de tous les niveaux dans des salles équipées de projecteurs et de 
tableaux blancs interactifs. Pour ces cours, hormis les enseignants locuteurs natifs, l'Institut embauche des enseignants monténégrins ayant plusieurs années d'expérience dans l'enseignement du FLE et titulaires du DALF C1 au minimum. Contrairement aux écoles, il recense de plus en plus d'apprenants inscrits aux cours de français.

Toutefois, malgré cette offre généreuse en matière culturelle et linguistique, l'État français ne semble plus être aussi intéressé qu'autrefois pour diffuser le français en dehors des portes de l'Institut. Les bourses destinées aux enseignants de FLE sont plus rares et les établissements scolaires du pays qui offrent du français ne sont plus soutenus en équipement technique, ni en supports pédagogiques.

Afin d'accroître l'intérêt pour l'apprentissage du français et d'augmenter sa popularité auprès des jeunes générations, l'Association des professeurs de français du Monténégro (APFM), fondée en 1998, organise de nombreuses activités, concours, festivals, colloques, etc. Regroupant tous les francophones et francophiles du pays, l'APFM compte de nos jours une centaine de membres dont 87, entre autres fonctions, assurent l'enseignement du français à tous les niveaux (72 enseignants au niveau primaire et secondaire et 15 enseignants au niveau universitaire).

Une recherche menée par l'APFM et que j'ai eu l'occasion de diriger, montre qu'en 2014/15, 8628 élèves avaient opté pour l'apprentissage du français au niveau primaire et secondaire, ce qui représente environ $8,6 \%$ de la totalité des effectifs d'élèves. Quant à l'Université, quelques 150 étudiants y suivent des cours de français, ce qui représente $0,75 \%$ du nombre total d'étudiants. ${ }^{4}$

\section{L'ENSEIGNANT DE FLE AU MONTÉNÉGRO}

\subsection{La formation à la profession}

Les enseignants de FLE, autrefois formés essentiellement dans les Universités de l'ex-Yougoslavie (Belgrade, Novi Sad, Priština, Sarajevo, Skoplje) sont depuis 2003 formés à l'Université du Monténégro 5 à la Faculté de philologie implantée dans la ville de Nikšić, au sein du département de Langue et littérature françaises (dorénavant département de français). C'est en 1999/2000 que le ministère français des Affaires étrangères avait confié à l'Université de Tours la gestion d'un Cocoop ${ }^{6}$ ayant pour objectif la mise en place d'un département de français à l'Université du Monténégro. Ce projet a permis de financer la formation de cinq doctorants monténégrins à Tours qui, embauchés par la suite par l'Université du pays, ont concouru à la construction du département en question, aux normes européennes.

\footnotetext{
${ }^{4}$ Le français est au niveau universitaire enseigné à la Faculté de philologie (Nikšić), à la Faculté de tourisme et d'hôtellerie (Kotor), ainsi qu'à la Faculté d'économie (Podgorica), laquelle réalise un programme de formation avec l'Université Sophia Antipolis de Nice.

${ }^{5}$ L'Université du Monténégro fondée en 1974, est le seul établissement universitaire d’État. Deux universités privées ont également ouvert leurs portes à la suite de la reconnaissance de l'indépendance du pays, mais elles ne disposent pas de département formant des enseignants de FLE.

${ }^{6}$ Projet subventionné visant le renforcement de la langue et de la culture française dans les pays de l'Est et du Sud-Est de l'Europe.
} 
De nos jours, la formation initiale des futurs enseignants de FLE consiste en un cursus universitaire de quatre ans (Licence + spécialisation) lors duquel, parallèlement à la langue, la littérature et la civilisation françaises y sont enseignées. L'initiation à la didactique du FLE, ainsi que l'initiation professionnelle ne sont au programme de ce cursus qu'en quatrième année. Outre le nombre d'heures considérable consacré aux savoirs grammaticaux, à la traduction et à la littérature, ce programme est pratiquement dépourvu de cours destinés au développement des compétences communicatives des étudiants en langue française. En fait, un seul cours de 45 minutes par semaine est consacré aux activités de communication avec un lecteur FLE-locuteur natif.

Mentionnons que la conception des plans et des programmes d'enseignement du département de français de l'Université du Monténégro a été attribuée à des professeurs - experts de l'ex-Yougoslavie à une époque où les étudiants qui s'orientaient vers des études de FLE disposaient d'une meilleure compétence communicative de la langue française. De plus, aucun justificatif du niveau de français n'est demandé lors de l'inscription et de plus en plus d'inscrits ont un niveau du CECRL inférieur à B1, voire A2. De ce fait, nous estimons que l'itinéraire de leur formation initiale, inspiré par ceux des facultés de la région et créé à une époque où le statut du français était plus favorisé dans les systèmes éducatifs, nécessite impérativement une révision considérable et doit être promptement réactualisé.

Tous les ans, grâce au soutien de l'Institut français de Podgorica et de l'Institut de l'Éducation nationale, l'APFM organise un colloque destiné à la formation continue des enseignants de FLE, certifié et reconnu officiellement par le ministère de l'Éducation nationale. À ce colloque, d'une durée de trois jours, interviennent conjointement des formateurs monténégrins et français. Toutefois, bien qu'il comble partiellement les lacunes des enseignants en matière de didactique et encourage les méthodes innovantes, le colloque n'est généralement suivi que par la moitié des enseignants de FLE actifs au Monténégro. Précisons que la formation continue est au Monténégro depuis 2013 obligatoire pour les enseignants qui désirent renouveler leur licence d'enseignement, valide cinq ans. Il est indispensable d'avoir suivi en cinq ans seize heures de formation « en matière prioritaire » et " huit heures d'autres contenus destinés à la formation continue des enseignants $\gg{ }^{7}$

Le recrutement des enseignants de FLE s'effectue au Monténégro par voie de concours. Un diplôme d'enseignant de FLE (obtenu après quatre années de cursus universitaire), ainsi qu'un stage de neuf mois effectué au sein d'un établissement scolaire de niveau primaire ou secondaire suivi par des examens (mémoire, épreuves théorique et pratique) sont indispensables afin d'être titularisé dans le domaine de l'enseignement public. Il n'existe pas de recommandations pour les futurs enseignants de langues étrangères de passer un certain temps dans le pays de la langue cible avant d'obtenir le diplôme d'enseignant.

L'évaluation des enseignants engagés dans le système scolaire est assurée par l'inspection générale du ministère de l'Éducation nationale, en l'occurrence par un inspecteur de français qui rend visite aux établissements scolaires, à tous les niveaux.

\footnotetext{
${ }^{7}$ Pravilnik o bližim uslovima, načinu i postupku izdavanja i obnavljanja dozvole za rad nastavniku, direktoru i pomoćniku direktora obrazovno-vaspitne ustanove, Službeni list Crne Gore, br.23/14 od 30.05.2014.
} 


\subsection{Les difficultés du métier}

La profession d'enseignant est un métier qui relève du défi et demande du dévouement. Pour Christophe Hélou et Françoise Lantheaume, les difficultés du travail des enseignants représentent une part consécutive du métier et sont liées essentiellement aux problèmes de l'intérêt des élèves, aux tensions entre sphères personnelle et professionnelle, et finalement à la difficulté de définir ce qu'est un bon travail (Hélou, 2008 : 66). Au cas où l'enseignant ne parviendrait pas à capter l'attention des élèves, la discipline dans la classe deviendrait difficile à contrôler, les élèves ne progresseraient pas et le cours perdrait du sens. Etant donné que les élèves deviennent de plus en plus difficiles à motiver, ce qui est en partie dû à un rapport plus critique aux savoirs, les enseignants dépensent de plus en plus d'énergie et de temps à préparer des activités pour leurs cours afin d'intéresser les élèves. Hélou et Lantheaume soulignent que trouver un bon texte, un bon thème, une bonne activité est devenu une réelle obsession professionnelle des enseignants (Hélou, 2008 : 67).

Les enseignants que la société a tendance à considérer comme des citoyens privilégiés à cause de leur temps de travail et des vacances scolaires prolongées, sont en fait sous la pression permanente du travail. Au temps de service qui se déroule en classe en présence d'élèves s'ajoute le temps destiné à la préparation des cours, à la correction des copies, aux réunions, aux conseils de classe, ainsi qu'aux tâches administratives de plus en plus exigeantes auxquelles tous les enseignants au niveau européen sont soumis.

De plus, les études montrent que le risque de présenter des troubles vocaux est environ trois fois plus élevé chez les enseignants que dans la population générale (Robieux, 2014 : 31 ) et que l'utilisation prolongée de la voix entraîne inévitablement un phénomène de fatigue vocale. Camille Robieux et Antoine Giovanni soulignent que la voix didactique a en général une intensité sonore et une hauteur tonale plus élevée que la voix d'expression simple et que si l'enseignant dépasse les limites physiologiques de son appareil vocal, une inflammation et d'autres troubles de la voix peuvent faire apparaitre des lésions sur les cordes vocales.

$\mathrm{Vu}$ le lien étroit entre voix, paroles et langues, les enseignants de langues étrangères ont un problème supplémentaire car un changement vocal translinguistique est susceptible de les gêner (Pillot-Loiseau, 2014 : 65). En analysant le geste vocal chez les enseignants de langues, Claire Pillot Loiseau constate qu'un forçage articulatoire est ressenti sous la forme d'une voix fatiguée, forcée et cassée lorsque les enseignants passent d'une langue à autre pendant leurs cours. Selon les résultats de ses recherches, le fait d'utiliser simultanément deux langues en situation d'enseignement peut aboutir à une gêne vocale ressentie qui est d'autant plus importante que les enseignants alternent au quotidien l'utilisation des deux langues qu'ils parlent (Pillot-Loiseau, $2014: 64$ ).

$\mathrm{Au}$ Monténégro, le métier d'enseignant au niveau primaire ou secondaire figure parmi les professions les moins bien rémunérées parmi celles financées par le budget national nécessitant un niveau de formation de quatre années à l'université. Le salaire des enseignants est souvent inférieur ou à peine égal au salaire mensuel moyen du pays qui correspond actuellement à 511 euros. $^{8}$

L'enseignant, à tous les niveaux d'études, enseigne la langue étrangère dans une classe dont les effectifs ne sont pas réduits et peuvent dépasser trente à trente-trois élèves, ce qui

\footnotetext{
${ }^{8}$ http://www.monstat.org/cg/novosti.php?id=2334
} 
est le maximum prescrit par la loi. Il est évident qu'il est difficile de faire progresser les apprenants dans de telles conditions, particulièrement lorsqu'il s'agit du français, qui appartient à une famille de langue différente de celle des élèves, ${ }^{9}$ dont ils sont éloignés géographiquement et qu'ils ont du mal à entendre à l'extérieur de la salle de classe. Mentionnons qu'un certain nombre de pays de l'Europe de l'Est qui font partie de l'UE recommandent un effectif réduit pour les cours de langue telle que la République tchèque et la Pologne (24 élèves), la Slovaquie (17 élèves) et la Lituanie (20 élèves), alors qu'en Hongrie les cours de langue se donnent souvent en petits groupes. ${ }^{10}$

D'autre part, un grand nombre d'écoles du pays manquent de matériel audio-visuel et informatique pour la réalisation des cours de langues étrangères et il n'est pas rare qu'un magnétophone ou un lecteur CD ne soit pas mis à disposition des enseignants. D'ailleurs, dans le questionnaire présenté ultérieurement, c'est le manque d'équipement dans les salles de classe qui représente la principale difficulté liée au métier d'enseignant de FLE.

L'intérêt pour l'apprentissage du français étant en baisse, les écoles embauchent de moins en moins d'enseignants et ceux qui ont gardé leurs postes sont souvent les seuls enseignants de FLE au sein de leurs établissements. De ce fait, inquiets pour leur avenir, ils éprouvent souvent un sentiment de rejet et d'isolement par rapport aux enseignants d'anglais, ou à ceux d'autres langues étrangères qui semblent avoir un meilleur statut. D'autre part, les enseignants de différentes langues ne se reconnaissent souvent pas comme faisant partie de la même profession et cette concurrence entre les langues semble souvent les séparer au lieu de les unir (Kelly, $2014: 401$ ).

\section{L'ENSEIGNANT dE FLE VIS-À-VIS DE SON MÉTIER}

\subsection{Le rapport des enseignants à l'exercice de leur formation}

Dans le souci d'intervenir dans la réalisation de nouveaux programmes à l'Université et de recadrer les voies de formation du département de français, nous avons d'abord voulu identifier le rapport des enseignants vis-à-vis de leur formation. Il s'agissait de rechercher chez les enseignants des informations et des expériences sur leur propre vie d'étudiant, avant qu'ils soient devenus enseignants de FLE eux-mêmes, en essayant de mettre en relief les éléments qu'ils privilégiaient dans leur cursus universitaire et qu'ils jugeaient utiles pour l'exercice de leur profession.

De ce fait, en juin 2016, lors d'un stage de formation continue se déroulant à Budva sur le littoral monténégrin, nous avons procédé à une enquête auprès de trente-six enseignants de FLE. Ce volume d'échantillon correspondait à la moitié du nombre total des enseignants de FLE actifs au Monténégro. Le matériel recueilli se constituait de réflexions personnelles d'enseignants exerçant leur profession dans les écoles primaires (dans les classes de $7^{\text {ème }}$, $8^{\text {ème }}$ et $9^{\text {ème }}$, ce qui correspond à l'âge des élèves de 12,13 et 14 ans) et secondaire (lycée d'enseignement général et lycées techniques spécialisés en tourisme, hôtellerie et économie, là où le français est enseigné pendant quatre ans), quels que soient leur âge, sexe, lieu de

\footnotetext{
${ }^{9}$ La langue officielle du pays est le monténégrin. Langue d'origine slave, le monténégrin est similaire au serbe, au croate et au bosniaque, regroupés autrefois sous un même nom, le serbo-croate.

${ }^{10}$ Chiffres clés de l'enseignement des langues à l'école en Europe 2012, Eurydice, Eurostat, p.122.
} 
travail ou de formation. L'analyse des données du questionnaire distribué aux enseignants (cf. annexe 1) a été de type qualitatif et s'appuyait sur la technique d'analyse textuelle du contenu, à partir de laquelle nous avons procédé à une catégorisation des idées exprimées.

Notre hypothèse de départ était qu'un niveau du CECRL inférieur à $\mathrm{C} 1$, voire B2 observé particulièrement chez les jeunes enseignants diplômés (auxquels le français a été parfois enseigné comme troisième langue étrangère à l'âge scolaire) contribuait à une certaine insécurité linguistique au sein de la classe, ce qui pouvait susciter un blocage vis-à-vis des élèves dans le processus d'enseignement. Pour la même raison, ces enseignants-là pouvaient ressentir une certaine résistance à l'égard de leurs formateurs français et de leurs collègues, lors des colloques de formation continue auxquels ils ne participaient que périodiquement. Mentionnons en rapport avec cette problématique qu'une recherche, effectuée récemment en Turquie auprès de 998 étudiants et dont l'objectif était de déterminer les caractéristiques de l'enseignant efficace en langues étrangères, a montré que la qualité de la prononciation, ainsi que les savoir-faire en matière de production orale figuraient parmi les qualités les plus appréciées en savoirs spécifiques liés aux contenus de la discipline et à la pédagogie des enseignants de langue (Çelik, 2013 : 293).

L'analyse du contenu des questions ouvertes posées aux enseignants de FLE fait apparaître certains énoncés et explications plus fréquemment que d'autres. Notamment, aux questions :

Qu'est-ce que vous avez apprécié le plus lors de votre formation initiale, la moitié $\mathrm{du}$ nombre total d'enseignants-informateurs a répondu qu'il s'agissait des cours de production orale assurés par un lecteur FLE-locuteur natif, alors que les cours magistraux de langue (mentionnés six fois), de littérature et de civilisation française (mentionnés cinq fois) ont été évoqués beaucoup plus rarement.

Qu'est-ce que vous changeriez dans les programmes d'enseignement au département de français, plus de la moitié des enseignants de FLE (dix-neuf réponses) a mentionné qu'il était absolument indispensable d'augmenter le nombre d'heures de cours attribués aux activités animées par un lecteur FLE-locuteur natif et d'accorder aux étudiants davantage de possibilités afin de suivre des programmes de formation pour l'enseignement du FLE en France. L'insuffisance du nombre d'heures de cours destinées à l'initiation à la didactique de FLE a été mentionnée par cinq enseignants, mais avec le constat que le colloque annuel de la formation continue organisé par l'APFM proposait des pistes et des méthodes innovantes.

Afin de répondre à la question concernant les contenus des programmes de formation continue organisés par l'APFM, les enseignants devaient cocher, selon le degré d'utilité, les activités qu'ils jugeaient plus ou moins efficaces pour la réussite de leurs cours. Sur trente enseignants ayant répondu à cette question, dix-neuf considéraient qu'un mariage de différentes activités leur semblait le plus efficace, alors que neuf enseignants jugeaient qu'il serait utile de favoriser davantage les activités liées au développement de la compétence langagière orale chez les apprenants. Deux enseignants seulement considéraient qu'une formation plus académique en matière de didactique du FLE leur semblait la plus utile.

\subsection{Le rapport des enseignants à l'exercice de leur profession}

Face à la baisse de motivation envers la profession d'enseignant, un autre objectif de notre recherche était d'explorer les représentations des enseignants de FLE vis-à-vis de leur métier afin de pouvoir déterminer la portée du lien entre l'enseignant et l'apprenant. Aux questions posées: 
Quel est le mot qui vous associe le plus au métier d'enseignant, les énoncés à connotation affective sont ceux qui se répètent le plus souvent. Le mot amour a été mentionné six fois, échange avec les enfants huit fois, compréhension deux fois, enthousiasme deux fois, mission trois fois. Les termes en rapport avec l'éducation ou l'enseignement en général ont été cités plus rarement. Outre les termes transmissions de savoirs et apprentissage, mentionnés cinq fois, les mots tels qu'école, élèves, cours, salle de classe, répétition ont été relevés une seule fois par enseignant-informateur.

Quels sont, selon vous, les bons côtés liés au métier d'enseignant de FLE, de nouveau, ce sont les réponses d'ordre affectif et émotionnel qui ont été le plus souvent relevées. Parmi les réponses les plus fréquentes, mentionnons : amour envers la langue, travail avec les jeunes générations, ainsi que sentiment de satisfaction lorsque les élèves continuent d'apprendre le français plus tard dans leur vie. Parmi les réponses en rapport avec le métier d'enseignant de langue en général, mentionnons : vacances scolaires, horaires avantageux et possibilité de séjourner en France.

Quelles sont les principales difficultés liées à votre métier? La majorité des enseignants monténégrins estiment que les conditions dans lesquelles ils exercent leur profession sont loin d'être convenables. Ils mentionnent le manque de matériel technique, informatique et celui des manuels de FLE, ainsi que des salles de classe inconvenablement équipées. Six enseignants considèrent leurs classes surchargées, alors que pour trois enseignants questionnés le volume horaire consacré à l'apprentissage du FLE est insuffisant. Une baisse de la motivation de la part des élèves a été mentionnée par trois enseignants-informateurs, alors que deux enseignants estiment que les heures de cours dédiées à l'enseignement du FLE ne devraient pas être les dernières placées dans l'emploi du temps des élèves.

\subsection{L'enseignant confronté au (dés)intérêt à l'égard du FLE}

Dans l'intention de mieux comprendre le désintérêt envers l'apprentissage du FLE dans les écoles monténégrines, nous avons interrogé en mars 2013, à travers un questionnaire, 304 élèves en classe de $6^{\text {ème }}(11$ ans) à l'âge où ils s'exprimaient sur le choix de la deuxième langue étrangère, décision qui allait orienter majoritairement leur apprentissage ultérieur des langues étrangères. Il était question d'élèves qui fréquentaient dix écoles situées dans les régions touristiques du littoral monténégrin et dans l'ancienne capitale historique Cetinje, là où le français est de nos jours le moins enseigné au Monténégro. Afin de repérer l'image relative à la langue française deux questions étaient posées aux collégiens : Comment décririez-vous la langue française? et Pourquoi choisiriez-vous cette langue? (Jovanović, 2013 : 410).

Selon les résultats de ce questionnaire, la plupart des réponses des élèves $(79 \%)$ concernant le choix ou le rejet du français se référait à la sonorité de la langue française (langue agréable à entendre ou à parler, ou au contraire, celle d'une langue dont on n'aime pas du tout le corps sonore) et dont l'image a été le plus souvent représentée par la catégorie de l'amour ou du mépris des personnes qui parlent français. Les exemples d'énoncés tels que $J e$ hais le français. Leur accent m'énerve /J'aime leur accent/ J'en suis tombé amoureuse. Dans cette langue il y a beaucoup d'amour montrent que les affects (amour/ haine/ plaisir/ refus) liés à l'image de la voix sont évidents (cf. annexe 2).

D'autre part, les résultats de cette étude dévoilent que $48 \%$ d'élèves ont été influencés par leurs parents dans le choix de la deuxième langue étrangère. Les représentations des 
parents envers les langues, qu'elles soient positives ou négatives, peuvent facilement être infusées à leurs enfants et ils peuvent garder cette attitude transmise pendant longtemps (Jovanović, $2013: 415$ ).

\section{Proposition d'AJustement de L'itineraire de FORMation initiale des ENSEIGNANTS DE FLE}

L'étude qui vient d'être exposée pourrait nous être utile pour le réajustement du programme de formation du département de français qui est actuellement composé majoritairement de disciplines dont les contenus s'appuient sur des savoirs académiques. Depuis l'ouverture du département à l'Université du Monténégro, les programmes et les contenus ont été plusieurs fois revus mais n'ont pas subi de vraies modifications.

De manière traditionnelle, les domaines liés aux études du FLE sont déclinés en deux catégories : linguistique et littérature. Le nombre d'heures destinées aux cours magistraux domine dans les programmes en vigueur, alors que six heures de cours de 45 minutes seule-

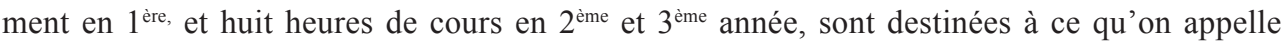
les travaux dirigés de la discipline nommée Français contemporain. Au cours de ces heures-là, les étudiants sont censés se familiariser à la traduction à l'écrit et à l'analyse grammaticale, et pendant une seule heure de cours animée par un lecteur FLE-locuteur natif, se consacrer aux activités servant essentiellement à l'acquisition de compétences communicatives orales.

De ce fait, nous considérons que la réforme curriculaire, actuellement en cours à l'Université du Monténégro, devrait tenir compte du rejet des anciens étudiants, devenus enseignants, que nous avons questionnés, envers les programmes qui n'aspirent pas suffisamment au développement de la capacité communicative en langue étrangère et augmenter considérablement le nombre d'heures de cours consacrées au développement de compétences orales.

D'autre part, les résultats de notre questionnaire dévoilent que dans leur pratique, les enseignants privilégient le caractère affectif lié à l'enseignement des langues étrangères auquel il faudrait davantage sensibiliser les étudiants de FLE en formation initiale. Une atmosphère de confiance est indispensable afin que les apprenants puissent s'épanouir (Bogaards, 1991 : 124). La portée de l'émotion dans l'apprentissage des langues demande plus de connaissances en matière de psychologie de l'individu et de psychanalyse (Aguilar Rio, 2014 : 345), domaines également insuffisamment abordés en formation initiale au Monténégro.

Dans les réponses des collégiens questionnés sur les représentations et le choix de la langue française domine l'image d'une langue dont on aime ou on n'aime pas du tout le corps sonore. De ce fait, nous considérons qu'il serait envisageable d'augmenter la motivation des apprenants en formant les futurs enseignants de FLE à se concentrer plus intensivement en classe sur un travail visant la compétence de production orale et les sons de la langue française.

Et finalement, les collégiens étant en grande partie influencés par leurs parents dans le choix de la langue étrangère, les enseignants de FLE devraient à l'avenir davantage sensibiliser les apprenants, ainsi que leurs parents, sur l'utilité de la maîtrise du français, leur permettant de trouver plus facilement un emploi, notamment dans le secteur du tourisme. 


\section{Conclusion}

La profession d'enseignant de FLE au Monténégro n'a plus le prestige d'autrefois et ceux qui se décident à l'exercer doivent lutter fermement pour un meilleur statut de la langue française, fortement concurrencé par l'anglais, langue véhiculaire à l'échelle internationale d'une part, et l'italien et le russe traditionnellement implantés d'autre part.

Estimant leur métier comme essentiellement relationnel, les enseignants de FLE tâchent de compenser le sentiment d'isolement qu'ils éprouvent au sein de leurs écoles, où ils sont souvent les seuls responsables de la matière, ainsi que celui de la sous-estimation matérielle de leur profession, par une vie associative dynamique conduite par l'APFM et la participation aux activités proposées par l'Institut français de la capitale monténégrine Podgorica.

L'aspect phonique d'une langue, aussi bien que sa qualité de prononciation, figurant parmi les éléments ayant le plus d'impacts sur les apprenants de FLE, il serait intéressant d'en tirer profit et de réfléchir à former dans l'avenir des enseignants de FLE munis d'une compétence orale de meilleure qualité et d'une base linguistique plus solide.

D'autre part, il serait indispensable de situer les futurs enseignants de FLE dans la réalité éducative et linguistique du Monténégro dans laquelle le français n'a plus sa place d'autrefois, mais figure dans le contexte économique du pays parmi les langues les plus demandées dans le secteur du tourisme, actuellement le plus grand employeur monténégrin. C'est en renforçant les liens avec le monde du travail que les enseignants de FLE doivent attirer l'intérêt des chefs d'établissements et celui des décideurs au niveau national qui orientent la politique linguistique et éducative du pays.

Et pour finir, re-citons Michael Kelly qui nous rappelle que l'avenir de l'enseignement des langues dépend de ceux qui la pratiquent, donc des enseignants (Kelly, 2014 : 399).

\section{RÉFÉRENCES BIBLIOGRAPHIQUES}

Aguilar Río, J.I. (2014). "Le développement personnel comme processus de formation des enseignants de langue(s)", in M.Causa, S.Galligani et M.Vlad (eds.), Formation et pratiques enseignantes en contextes pluriels, Paris: Riveneuve, 333-354.

Andrijašević, Ž. et Rastoder, Š. (2000). Histoire du Monténégro. Coopération Luxembourg-Monténégro.

Bogaards, P. (1991). Aptitude et affectivité dans l'apprentissage des langues étrangères. Paris: Hatier/Didier.

Bokan, M. (1961). "Ruski i engleski prioritetni u nastavi", in Prosvjetni rad, VIII, $16: 2$

Čakić, K. (1997). "Francusko zabavište na Cetinju", in Cetinjski list, 406 : 9.

Çelik, S., Arikan A. and Caner M. (2013). "In the Eyes of Turkish EFL Learners: What Makes an Effective Foreign Language Teacher?", in Porta Linguarum, 20: 287-297, available from : http://www.ugr.es/ portalin/articulos/PL\%20issue20\%20Jun2013.htm, accessed 20 June, 2016

Chiss, J.L.(2007). "Didactique des langues et histoire(s) de formation(s)", in Recherche et applications 41: 182-191.

Hélou C. et Lantheaume F. (2008). "Les difficultés au travail des enseignants - exception ou part consécutive du métier", in Recherche et formation 57: 65-78. 
Jovanović I., Milivojević A. (2007). "Nastava stranih jezika i razvoj turizma i hotelijerstva u Crnoj Gori", in Hotellink, 9-10: 897-898.

Jovanović, I., Milivojević I. (2013). "La situation et l'image du français dans le système éducatif monténégrin", in DEAF 2 Actes du IIè colloque international, Faculté des Lettres et des Arts, Université de Kragujevac: 403-417.

Jovanović, I. (2016). Langue et culture françaises au Monténégro (1830-1914), Podgorica: UCG. Laval, E. (1920). "O nastavi francuskog jezika", in Prosvetni glasnik, XXXXVI, 3 : 157-158.

Kapetanović, M. (1961). "Aktuelni problemi u nastavi stranih jezika" in Prosvjetni rad, VIII, $16: 3$

Kelly, M. (2014) "Que peut l'Europe? Comment les décideurs peuvent mieux soutenir l'enseignement des langues", in M.Causa, S.Galligani et M.Vlad (eds.), Formation et pratiques enseignantes en contextes pluriels, Paris: Riveneuve, 399-401.

Lee, K.N. (2011). "L'enseignement du français au lycée en Corée : sélection et orientation des contenus et leurs limites", in Recherche et applications 49: 125-136.

Pillot-Loiseau, C.(2014). "Geste vocal, voix et enseignement des langues", in M. Tellier et L. Cadet (eds.), Le corps et la voix de l'enseignant: théorie et pratique, Paris: Maison des langues, 51-66.

Robieux, C. and Giovanni A. (2014). "Les troubles de la voix chez l'enseignant", in M. Tellier et L.Cadet (eds.), Le corps et la voix de l'enseignant: théorie et pratique, Paris: Maison des langues, 29-37.

\section{Références liées aux documents institutionnels :}

Chiffres clés de l'enseignement des langues à l'école en Europe 2012, Eurydice, Eurostat, availible from http://eacea.ec.europa.eu/education/eurydice/documents/key_data_series/143fr. pdf, accessed 15 june 2016.

Nastavni planovi i programi. (1934). Glasnik JPD, XV, 4 : 345-346.

Podaci o pohadjanju stranih jezika u osnovnim i srednjim školama za školsku 2011/2012. Ministarstvo prosvjete, Odjeljenje za informaciono-komunikacione tehnologije.

Pravilnik o bližim uslovima, načinu i postupku izdavanja i obnavljanja dozvole za rad nastavniku, direktoru i pomoćniku direktora obrazovno-vaspitne ustanove, in Službeni list Crne Gore, br.23/14 od 30.05.2014, availible from http://www.sindikatprosvjete.me/dokumenta/propisi/ Pravilnik o licenciranju nastavnika.doc, accessed 15 June 2016.

http://eacea.ec.europa.eu/education/eurydice/documents/key_data_series/143fr.pdf , accessed 15 june 2016.

http://gerflint.fr/Base/Essais_francophones/essais_francophones_3_livre_blanc_fipf-1.pdf, accessed 20 june 2016.

http://www.google.me/search?q=14.+Pravilnik + o+bližim + uslovima $\% 2 C+$ načinu $+\mathrm{i}+$ postupku+izdavanja $+\mathrm{i}+$ obnavljanja + dozvole $+\mathrm{za}+\mathrm{rad}+$ nastavniku $\% 2 \mathrm{C}+$ direktoru $+\mathrm{i}+$ pomoćniku + direktora + obrazovnovaspitne + ustanove $\% 2 \mathrm{C}+$ Ministarstvo + prosjete $\% 2 \mathrm{C}+$ Član $+6 \&$ oq $=14 .+$ Praviln$\mathrm{ik}+\mathrm{o}+$ bližim + uslovima $\% 2 \mathrm{C}+$ načinu $+\mathrm{i}+$ postupku+izdavanja $+\mathrm{i}+$ obnavljanja + dozvole $+\mathrm{za}+\mathrm{ra}-$ $\mathrm{d}+$ nastavniku $\% 2 \mathrm{C}+$ direktoru $+\mathrm{i}+$ pomoćniku+direktora + obrazovnovaspitne + ustanove $\% 2 \mathrm{C}+\mathrm{Mi}-$ nistarstvo + prosjete $\% 2 \mathrm{C}+\mathrm{C}$ lan $+6 \&$ aqs $=$ chrome..69i57.33794j0j4\&sourceid $=$ chrome $\& \mathrm{ie}=\mathrm{UTF}-8$ , accessed 25 june 2016.

http://www.monstat.org/cg/novosti.php?id=2334, accessed february 2016. 


\section{ANNEXE 1 \\ Questionnaire destiné aux enseignants de FLE au Monténégro}

\section{Le métier d’enseignant de FLE}

1. Quel terme ou mot vous associe le plus au métier d'enseignant de langue?

2. Quels sont, selon vous, les bons côtés liés au métier d'enseignant de FLE au Monténégro?....

3. Quelles sont les principales difficultés liées au métier d'enseignant de FLE au Monténégro?

4. Quels sont les facteurs qui pourraient contribuer à un plus grand interêt de la part des élèves envers l'appentissage du français au Monténégro?

\section{LA FORMATION D'ENSEIGNANT DE FLE}

1. Mentionner les cours et les activités que vous avez appreciés le plus lors de votre formation initiale?.

2. Qu'est-ce que vous changeriez dans le cursus de formation initiale des enseignants de FLE au Monténégro?

3. Suivez-vous régulièrement les stages de formation continue?

4. Cochez de 1 à $5(1=\min$., $5=$ max.) les activités que vous jugez utiles lors des stages de formation continue :

a. Formation axée sur la théorie de l'enseignement de FLE

b. Formation axée sur le développement des compétences communicatives liées à la production orale

c. Formation axée sur le développement des compétences communicatives liées à la production écrite

d. Formation axée sur les analyses des pratiques en classe de langue

e. Mélange de différentes activités

5. Avez-vous eu la possibilité de vous perfectionner en France ? Si, oui, mentionnez le lieu et la période. 


\section{ANNEXE 2 \\ Les attitudes des collégiens monténégrins vis-a vis de la langue française}

\begin{tabular}{|c|c|}
\hline $\begin{array}{l}\text { Les arguments contre l'apprentissage du } \\
\text { français }\end{array}$ & $\begin{array}{l}\text { Les arguments en faveur de l'apprentissage } \\
\text { du français }\end{array}$ \\
\hline $\begin{array}{l}\text { - C'est très difficile de prononcer le } \\
\text { français. Cette langue est incom- } \\
\text { préhensible. } \\
\text { - C'est une langue bête, difficile à } \\
\text { prononcer } \\
\text { - Le français est ennuyeux. Ses mots } \\
\text { sont difficiles à prononcer. } \\
\text { - Cette langue ne me plaît pas. La seule } \\
\text { chose qui me plaît dans cette langue } \\
\text { ce sont les chansons françaises. Rien } \\
\text { d'autre. } \\
\text { - Le français a ce « R » bizarre. Je ne } \\
\text { l'aime pas à cause de ce « R qui est } \\
\text { tout à fait différent de notre « R ». } \\
\text { - Le français, c'est la langue des chats. } \\
\text { Ils ronronnent comme les chats avec ce } \\
\text { " R » bizarre. Cela ne me plaît pas. } \\
\text { - Il est très difficile. Il a beaucoup de } \\
\text { mots irréfléchis. } \\
\text { - Je ne l'ai pas choisi. Je hais le français. } \\
\text { Leur accent m'énerve. } \\
\text { - Cette langue ne me plaît pas parce } \\
\text { que je dois casser ma langue pour la } \\
\text { prononcer et je dois faire des mimiques } \\
\text { bizarres. } \\
\text { Il y a beaucoup de mots étranges en } \\
\text { français. Je ne peux pas l'apprendre } \\
\text { car je dois changer de voix. } \\
\text { - C'est la langue la plus difficile. Mon } \\
\text { père dit que les Français aiment manger } \\
\text { les mots comme s'ils avaient toujours } \\
\text { faim tandis que les Italiens prononcent } \\
\text { clairement les mots. } \\
\text { - Le français est très différent par rapport } \\
\text { aux autres langues et particulièrement } \\
\text { bizarre. Je ne l'ai pas choisi. J'ai } \\
\text { l'impression que les Français se donnent } \\
\text { de l'importance lorsqu'ils le parlent. } \\
\text { - Je ne l'ai pas choisi parce que je n'aime } \\
\text { pas leur débit. Ils parlent vite. } \\
\text { - }\end{array}$ & $\begin{array}{l}\text { - C'est la lettre « R » qui est très sympathique. } \\
\text { - Le français est beau car il est unique. } \\
\text { - C'est une langue qui repose la gorge lorsque } \\
\text { tu la prononces. } \\
\text { - C'est la langue des dames. Une vieille langue. } \\
\text { Je l'ai choisie pour ces raisons. } \\
\text { - C'est une langue qui a un bel accent. C'est } \\
\text { la langue de l'amour et de la beauté de } \\
\text { l'esprit humain. } \\
\text { - J'ai choisi cette langue car j'aimerais la } \\
\text { parler couramment un jour. J'aimerais être } \\
\text { comme les Français. Les Français ont une } \\
\text { culture riche. } \\
\text { - Le son « J » est dominant dans cette langue } \\
\text { c'est pourquoi dans cette langue on peut } \\
\text { draguer les filles. } \\
\text { - Elle sonne bien. Elle est remarquable. } \\
\text { - Le français me plaît. Les Français sont des } \\
\text { gens pleins d'émotions. Ils sourient toujours. } \\
\text { Quand j'étais chez ma tante en France, j'ai vu } \\
\text { que les Français se font très souvent la bise. } \\
\text { - Les lettres françaises sont douces tandis que } \\
\text { les lettres des autres langues sont plutôt dures. } \\
\text { C'est la raison pour laquelle je l'ai choisie. } \\
\text { - J'aime leur accent. } \\
\text { - La langue française est une langue très } \\
\text { élégante et féminine, c'est pourquoi je l'ai } \\
\text { choisie. } \\
\text { - Il me plaît car il a une prononciation bizarre, } \\
\text { c'est pourquoi il est unique. Je l'ai choisi à } \\
\text { cause de cela. Rares sont ceux qui peuvent } \\
\text { l'apprendre. } \\
\text { - Les mots de français sont beaux ainsi que } \\
\text { leur système vocal. } \\
\text { - Dans cette langue, il y a beaucoup d'amour. } \\
\text { J'aime les Français. } \\
\text { - C'est une langue magnifique qui vient d'un } \\
\text { pays magnifique. } \\
\text { - J'ai choisi le français car ma grand-mère } \\
\text { m'apprenait des mots français. J'aime les } \\
\text { prononcer. }\end{array}$ \\
\hline
\end{tabular}

